CLINICAL STUDY

\title{
Prevalence and demographic features of childhood growth hormone deficiency in Belgium during the period 1986-2001
}

M Thomas $^{1}$, G Massa ${ }^{1,2}$, M Craen $^{3}$, F de Zegher ${ }^{4}$, J P Bourguignon ${ }^{5}$, C Heinrichs ${ }^{2}$, J De Schepper ${ }^{6}$, M Du Caju $^{7}$, G Thiry-Counson ${ }^{9}$ and $\mathrm{M}_{\text {Maes }}^{8}$

${ }^{1}$ Belgian Study Group for Paediatric Endocrinology, and Departments of Paediatrics of the Universities of ${ }^{2}$ Bruxelles, ${ }^{3}$ Gent, ${ }^{4}$ Leuven, ${ }^{5}$ Liège, ${ }^{6}$ Brussel, ${ }^{7}$ Antwerpen, ${ }^{8}$ Louvain, Belgium and ${ }^{9}$ Clinique de l'Espérance, Saint-Nicolas, Belgium

(Correspondence should be addressed to Marc Maes, Département de Pédiatrie, Cliniques Universitaires Saint-Luc, Avenue Hippocrate, 10, 1200 Bruxelles, Belgium; Email: maes@pedi.ucl.ac.be)

\begin{abstract}
Objective: Since the availability of recombinant human growth hormone (rhGH) all children with growth hormone deficiency (GHD) living in Belgium are offered rhGH treatment after approval by a peer-review board. In this study, we evaluated the prevalence and demographic features of childhood GHD in Belgium during the period 1986-2001 and we compared them with the data from other countries.

Methods: Diagnostic, demographic and baseline auxological data of 714 children diagnosed as having GHD between 1986 and 2001 were retrieved from the database of the Belgian Study Group for Paediatric Endocrinology.

Results: The prevalence of GHD in Belgium was estimated to be 1/5600. The origin of GHD was idiopathic (idGHD) in $41 \%$ of the patients, congenital (congGHD) in $20 \%$ and acquired (acqGHD) in $35 \%$. During the first 4 years (1986-1989) more patients were classified as idGHD; thereafter the distribution between the three aetiology groups did not change. In all groups, boys outnumbered girls but this preponderance was especially pronounced in congGHD patients (male:female $=4: 1$ ) with a central malformation that associates an anterior pituitary hypoplasia, a missing, fine or normal pituitary stalk and an ectopic posterior pituitary. Thirteen percent of the patients with idGHD, 50\% with congGHD and $52 \%$ with acqGHD had multiple pituitary deficiencies. Patients with congGHD were the youngest (mean \pm S.D. age: $6.5 \pm 4.7$ years) and were the shortest $(-3.0 \pm 1.3$ standard deviation score (SDS)) at the start of rhGH treatment. There was no time trend over the studied period for age and height at onset of GH therapy.

Conclusion: In Belgium, the prevalence of childhood GHD can be estimated as 1/5600 which is comparable to other recent surveys. The yearly number of new patients for the different aetiologies and the auxological parameters have remained relatively constant over the last 16 years.
\end{abstract}

European Journal of Endocrinology 151 67-72

\section{Introduction}

Growth hormone deficiency (GHD) is a relatively uncommon cause of growth retardation and short stature. The prevalence of childhood GHD reported in the literature diverges widely varying between 1/3480 and 1/30 000 children $(1-8)$.

In 1986, recombinant human growth hormone (rhGH) was licensed for use in Belgium and since then supplies of rhGH have been virtually unlimited. Growth hormone deficiency remains the primary indication for rhGH treatment and all children with GHD can be offered optimal treatment. Treatment with rhGH is reimbursed by the Belgian government, and until 1993, the use of rhGH was supervised by a ministerial commission of experts. After the dismissal of the ministerial commission, the paediatric endocrinologists belonging to the Belgian Study Group for Paediatric
Endocrinology (BSGPE) continued to peer-review the records of patient candidates for rhGH treatment before applying for its reimbursement.

In the present study, we evaluated the prevalence and described demographic features of childhood GHD in Belgium during the period 1986-2001, and we compared the results with those from other databases and other countries. The data in this report present a profile of what could be considered standard diagnostic practices in Belgium.

\section{Patients and methods}

In Belgium, the files of children who are candidates for treatment with rhGH are discussed by the members of the BSGPE using a systematic peer-reviewed process. The BSGPE includes the Belgian paediatric 
endocrinologists treating children with growth disorders, and represents the seven Belgian universities. An application for treatment with rhGH to be reimbursed by the government is only accepted after a consensus decision by the majority of the BSGPE members. Baseline and treatment auxological and biochemical data from the patients approved for rhGH treatment are stored in the database of the BSGPE. Signed informed consent forms are obtained from the parents and the patients if older than 12 years.

From the database of the BSGPE, the data of all children $(n=740)$ diagnosed with GHD between 1986 and 2001 were retrieved. The data of 26 patients were incomplete and they were not included in the analysis. GHD was defined by auxological and biological criteria: height velocity $<$ the 10 th or $<$ the 25 th centile for patients with idiopathic and organic GHD respectively, and peak serum GH levels, measured in the individual institution's laboratory, $<10 \mathrm{ng} / \mathrm{ml}$ during two stimulation tests, usually the glucagon stimulation test and the insulin tolerance test (9). Sex steroid priming was carried out for boys aged 10 years or older and for girls aged 9 years or older if they had a pubertal development $\leq$ Genital stage 2 or Breast stage 2, independently of bone age. In boys, $50 \mathrm{mg}$ testosterone propionate were administered intramuscularly $72 \mathrm{~h}$ before the GH stimulation test, and the girls received orally $50 \mu \mathrm{g}$ ethinyloestradiol during the three days before the test (9). GHD was considered as complete when the maximal GH values at the two tests were below $5 \mathrm{ng} / \mathrm{ml}$; GHD was considered as partial when the maximal $\mathrm{GH}$ values at the two tests were below $10 \mathrm{ng} / \mathrm{ml}$ with at least one above $5 \mathrm{ng} / \mathrm{ml}$ (9). Cranial imaging studies, with special emphasis on the hypothalamo-pituitary region, were performed in all patients using computed tomography (CT) scanning or magnetic resonance imaging (MRI). Before 1990, an MRI was performed in $29 \%$ of the patients with non-acquired GHD, 1990-1993 MRI was performed in $76 \%$ and $1994-1997$ in $89 \%$ of these patients. After $1998,98 \%$ of the patients were evaluated by MRI.

The data collected in this study included aetiology of GHD, gender, age and height at the start of rhGH treatment, birth weight, parental heights, and associated pituitary deficiencies. The aetiologies of GHD were classified according to the Kabi International Growth Study aetiology classification list (10). Patients were classified as idiopathic GHD (idGHD) when no anomaly could be detected after the performed investigations. Patients were considered as congenital GHD (congGHD) when a cerebral developmental anomaly was documented or when a genetic cause of GHD was found. Patients with a postnatally acquired cerebral anomaly were classified as acquired GHD (acqGHD). Height was measured with a Harpenden stadiometer and expressed as standard deviation score (SDS) using the Tanner 1966 reference data (11). Birth weight SDS was calculated using the Swedish reference data (12). The patients were considered as small for gestational age (SGA) if birth weight was below -2 SDS. Mid-parental height SDS was calculated as (father's height SDS + mother's height SDS)/ 2 .

The prevalence of GHD was calculated on the basis of the Belgian demographic data provided by the National Institute of Statistics (13). Results are expressed as means \pm S.D. or median (range) as indicated. Comparisons between the three aetiology groups were carried out with the chi-squared test, or by ANOVA or Kruskal-Wallis test. The Jonckheere-Terpstra nonparametric test was used to test for time trends for age and height at onset of $\mathrm{GH}$ therapy. A $P<0.05$ value was considered significant.

\section{Results}

\section{Prevalence of GHD}

For the studied period, 714 (442 boys) patients with documented GHD were retrieved from the database of the BSGPE. Yearly, between 29 and 66 (median 45) new GHD patients started treatment. In 2001, 386 GHD patients between 0 and 18 years were treated with rhGH. Consequently, for the Belgian population, counting about 2.2 million children younger than 18 years, a prevalence (95\% confidence interval) of GHD children of 18 (16-20) per 100000 (or 1/5600) was estimated.

\section{Aetiology and gender}

The aetiology of GHD and the gender of the patients are given in Table 1. Forty-one per cent of the patients were classified as idGHD, $20 \%$ as congGHD and 35\% as acqGHD; $4 \%$ of the patients had a defined syndrome with concomitant GHD. The aetiology of the acqGHD was due, for the majority of the patients (93\%), to cranial tumours and/or cerebral radiotherapy. Boys outnumbered girls in all groups. The preponderance of boys was especially pronounced in the patients with congGHD represented mainly by patients with a central malformation, most frequently the association of hypoplastic anterior pituitary, missing, fine or normal stalk and ectopic posterior pituitary (sex ratio male:female $=4$ ).

Figure 1 shows the 4-yearly evolution of new patients with GHD. During the first 4 years (19861989) more patients were classified as idGHD. The yearly number of new patients with congGHD and acqGHD remained relatively stable over the whole period.

\section{Severity of GHD and associated pituitary deficiencies}

Of the patients with idGHD, 37\% had complete GHD while $78 \%$ of the patients with congGHD and $62 \%$ of the patients with acqGHD were diagnosed as complete GHD $(P<0.001)$. 
Table 1 Aetiology of GHD. The numbers of patients in the congenital and the acquired GHD groups (both belonging to the organic GHD group) were further subdivided depending on their specific aetiology.

\begin{tabular}{|c|c|c|c|c|c|c|}
\hline \multirow{2}{*}{ Group } & \multicolumn{3}{|c|}{ Number of patients } & \multirow[b]{2}{*}{$\%$ of total } & \multicolumn{2}{|c|}{ Sex ratio $M: F$} \\
\hline & $\begin{array}{l}\text { No. } \\
\text { in group }\end{array}$ & $\begin{array}{l}\text { No. in first } \\
\text { subgroup }\end{array}$ & $\begin{array}{l}\text { No. in second } \\
\text { subgroup }\end{array}$ & & Group & Subgroup \\
\hline Idiopathic GHD & 296 & & & 41 & 1.5 & \\
\hline Congenital form & 142 & & & 20 & 3.0 & \\
\hline Genetic cause (Pit-1, GH1, HESX-1 defect) & & 3 & & & & \\
\hline Central malformation & & 136 & & & & \\
\hline $\mathrm{HME}^{1}$ & & & 101 & & & 4.0 \\
\hline Other ${ }^{2}$ & & & 35 & & & 1.9 \\
\hline Other $^{3}$ & & 3 & & & & \\
\hline Acquired GHD & 245 & & & 35 & 1.2 & \\
\hline Cranial tumour of the pituitary-hypothalamus area ${ }^{4}$ & & 77 & & & & \\
\hline Irradiated & & & 37 & & & \\
\hline Non-irradiated & & & 40 & & & \\
\hline $\begin{array}{l}\text { Cranial tumour distant from the } \\
\text { pituitary-hypothalamus area }\end{array}$ & & 119 & & & & \\
\hline Irradiated & & & 110 & & & \\
\hline Non-irradiated & & & 9 & & & \\
\hline Treatment for malignancy outside the cranium & & 31 & & & & \\
\hline Acute lymphatic leukaemia & & & 25 & & & \\
\hline Lymphoma and other tumours outside the cranium 6 & & & 6 & & & \\
\hline Other causes of acquired $\mathrm{GHD}^{7}$ & & 18 & & & & \\
\hline Syndromes with concomitant GHD & 31 & & & 4 & 2.9 & \\
\hline Total & 714 & & & & 1.6 & \\
\hline
\end{tabular}

${ }_{2}^{1}$ Hypoplastic anterior pituitary-missing stalk, fine stalk or normal stalk-ectopic posterior pituitary.

${ }^{2}$ Septo-optic dysplasia $(n=8)$, mid-line palatal cleft $(n=5)$, arachnoid cyst $(n=4)$, congenital hydrocephalus $(n=9)$, empty sella syndrome $(n=5)$, solitary central maxillary incisor syndrome $(n=1)$, schizencephalia $(n=1)$, encephaloclastic lesion $(n=1)$, Rathke's cyst $(n=1)$.

${ }^{3}$ Syndromes with congenital GHD: q18 deletion $(n=1), 46, X X / 46, X X$ del(20) (p122) $(n=1)$; congenital toxoplasmosis $(n=1)$.

${ }^{4}$ Craniopharyngioma $(n=56)$, germinoma $(n=12)$, adenoma $(n=5)$, chordoma $(n=1)$, choriocarcinoma $(n=1)$, hypothalamic tumour $(n=2)$.

${ }^{5}$ Medulloblastoma $(n=47)$, astrocytoma $(n=24)$, glioma $(n=15)$, ependymoma $(n=7)$, rhabdomyosarcoma $(n=12)$, osteosarcoma $(n=1)$, gangliocytoma $(n=2)$, glioblastoma $(n=1)$, meningioma $(n=1)$, mesencephalic tumour $(n=1)$, neurocytoma $(n=1)$, papilloma $(n=1)$, primitive neuroectodermal turmour $(n=1)$, retinoblastoma $(n=2)$, teratoma $(n=2)$, other $(n=1)$.

${ }^{6}$ Lymphoma $(n=4)$, neuroblastoma/TBI $(n=1)$, hepatoblastoma $(n=1)$.

${ }^{7}$ Head trauma $(n=8)$, histiocytosis $(n=6)$, enlarged stalk $(n=1)$, CNS infection $(n=1)$, Total body irradiation for thalassaemia major $(n=1)$, BlackfanDiamond $(n=1)$

Table 2 shows the associated pituitary deficiencies. Fifty per cent of the patients with congGHD and $52 \%$ with acqGHD had other hormonal deficiencies, while these were present only in 13\% of the patients with idGHD $(P<0.01)$. Thyrotrophin (TSH) deficiency was the most frequent pituitary deficiency associated with GHD. Patients with congGHD and acqGHD had an

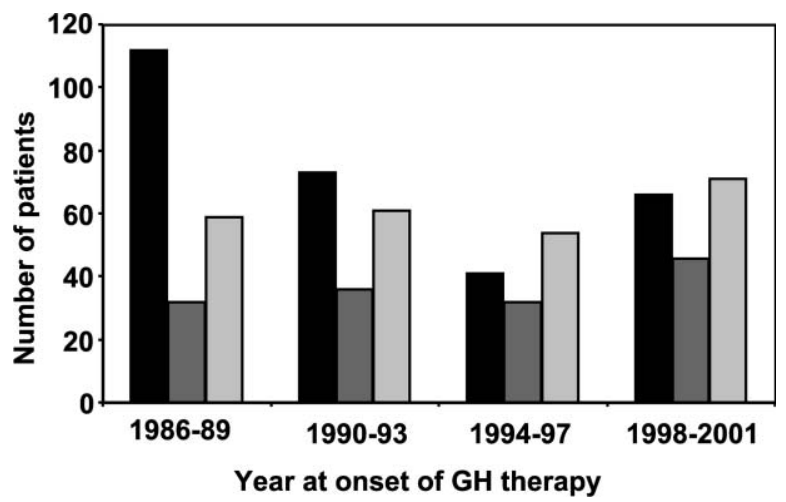

Figure 1 Four-yearly evolution of the number of new patients with idGHD (black bars), congGHD (dark grey bars) and acqGHD (light grey bars). adrenocorticotrophin (ACTH) deficiency more frequently than patients with idGHD $(P<0.01)$. In the three groups, about half of the patients with multiple pituitary deficiencies had luteinizing hormone/folliclestimulating hormone (LH/FSH) deficiency. More than half of the patients with multiple acqGHD had an associated antidiuretic (ADH) deficiency.

\section{Age and height at onset of therapy, birth weight and parental height}

Table 3 shows the age and height at onset of rhGH therapy, birth weight and parental height for the three aetiology groups. For the whole group of patients, rhGH therapy was started at the median age of 9.1 years (range $0.0-27.3$ years). The patients with congGHD were younger than the patients in the other groups $(P<0.001)$. In the three groups, no differences were found for the age at onset of GH therapy between patients with partial and complete GHD. In the patients with idGHD and congGHD rhGH treatment was started when a serious height deficit $(-2.8 \pm 0.9$ SDS and $-3.0 \pm 1.3$ SDS respectively) was already present. 
Table 2 Number of patients with multiple pituitary hormone deficiencies (MPHD) and distribution of other pituitary deficiencies for the different aetiologies.

\begin{tabular}{|c|c|c|c|c|c|c|}
\hline \multirow[b]{2}{*}{ Cause of GHD } & \multicolumn{2}{|c|}{ MPHD } & \multirow{2}{*}{$\underset{(\%)}{\text { TSH/MPHD }}$} & \multirow{2}{*}{$\begin{array}{c}\text { ACTH/MPHD } \\
(\%)\end{array}$} & \multirow{2}{*}{$\underset{(\%)}{\text { LH FSH/MPHD }}$} & \multirow{2}{*}{$\begin{array}{c}\text { ADH/MPHD } \\
(\%)\end{array}$} \\
\hline & $n$ & $\overline{\%}$ & & & & \\
\hline IdGHD $(n=296)$ & 38 & 13 & 82 & 34 & 48 & 5 \\
\hline CongGHD $(n=142)$ & 71 & 50 & 93 & 68 & 49 & 11 \\
\hline AcqGHD $(n=245)$ & 127 & 52 & 87 & 69 & 54 & 57 \\
\hline
\end{tabular}

Table 3 Age and height at onset of GH therapy, birth weight and mid-parental height (SDS) in the three aetiology groups (means \pm S.D.).

\begin{tabular}{lcccc}
\hline Group & $\begin{array}{c}\text { Age at } \\
\text { onset } \\
\text { (years) }\end{array}$ & $\begin{array}{c}\text { Height } \\
\text { at onset } \\
\text { (SDS) }\end{array}$ & $\begin{array}{c}\text { Birth } \\
\text { weight } \\
\text { (SDS) }\end{array}$ & $\begin{array}{r}\text { Mid-parental } \\
\text { height (SDS) }\end{array}$ \\
\hline IdGHD & $8.6 \pm 4.4$ & $-2.8 \pm 0.9$ & $-1.0 \pm 1.2$ & $-0.4 \pm 1.2$ \\
CongGHD & $6.5 \pm 4.7$ & $-3.0 \pm 1.3$ & $-0.7 \pm 1.0$ & $0.1 \pm 1.0$ \\
AcqGHD & $10.9 \pm 3.2$ & $-1.4 \pm 1.1$ & $-0.3 \pm 1.0$ & $0.2 \pm 0.9$ \\
\hline
\end{tabular}

Respectively, 90 and $86 \%$ of the patients with idGHD and congGHD were smaller than the 3rd percentile at the start of rhGH treatment, whereas only $35 \%$ of the patients with acqGHD were smaller than the 3rd percentile $(P<0.001)$. Age and height at onset of rhGH therapy did not change significantly during the studied period (data not shown).

Birth weight was significantly below the population mean $(P<0.001)$ in the patients with idGHD and congGHD. Respectively, 18, 11 and $4 \%$ of the patients with idGHD, congGHD and acqGHD were considered as SGA $(P<0.001)$. Mid-parental height SDS of the patients with congGHD and acqGHD was comparable to the general population. In contrast, patients with idGHD had a mid-parental height below the normal population $(P<0.001) ; 8 \%$ of the patients in this latter group had a mid-parental height below the 3rd percentile while this was the case in only 3 and $1 \%$ of the patients with congGHD and acqGHD respectively.

We did not find any major differences between boys and girls for the parameters detailed above except that in girls with congGHD and acqGHD, rhGH treatment was initiated at a younger age than in boys: 4.9 versus 7.2 years, and 10.2 versus 11.4 years $(P=0.003)$ respectively.

Figure 2 shows that $58 \%$ of the patients with congGHD started treatment before the age of 10 years; there was a bimodal distribution for the age at onset of rhGH therapy in children with idGHD, with a first peak around the age of 4-5 years and another peak around the age of 10-12 years. In the patients with acqGHD, the age at onset of therapy ranged between 8 and 15 years.

\section{Discussion}

Based on data from children diagnosed and treated for GHD by the members of the BSGPE, we estimated the a



b
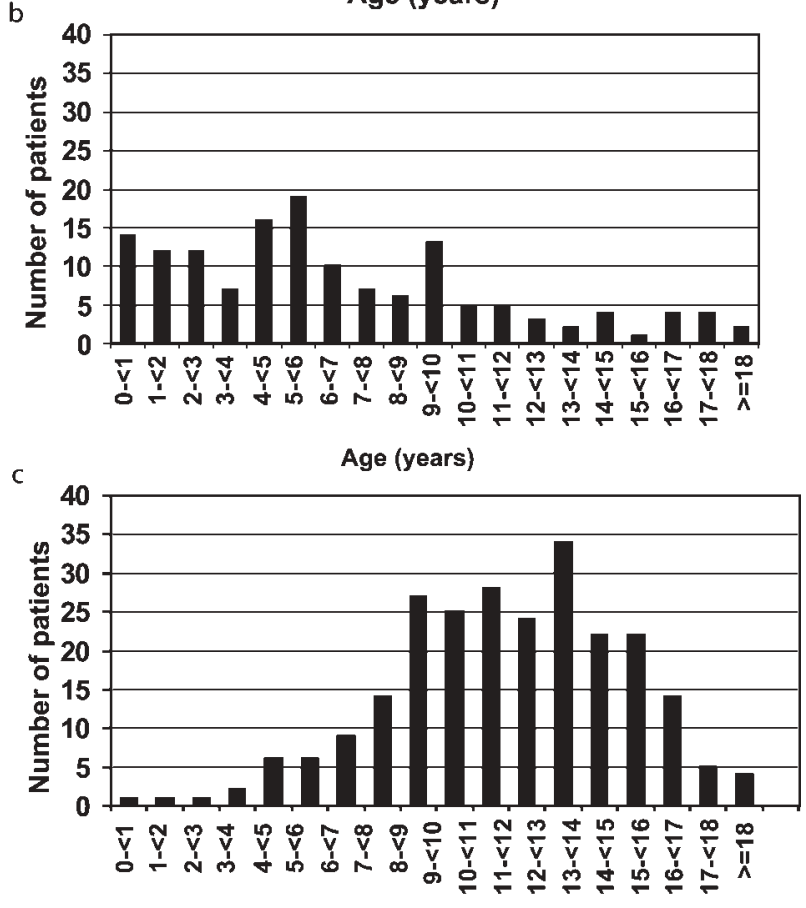

Age (years)

Figure 2 Age at onset of $\mathrm{GH}$ therapy in the three main groups: (a) idGHD, (b) congGHD, (c) acqGHD.

prevalence of childhood GHD in Belgium in 2001 to be $1 / 5600$. This number is comparable to the observations of the Dutch Growth Foundation (1/5000), using a comparable method to evaluate the prevalence of GHD (3). Our prevalence is somewhat lower than that reported in the United Kingdom (1/4018) (2) and in the state of Utah in the USA (1/3480) (1), 
while it is higher than in Australia (1/6757) (4), Japan (1/6797) (5) and China (1/8646) (6). As pointed out by Lindsay and colleagues (1), the true prevalence of GHD can only be derived from large-scale screening studies. In their survey evaluating 114881 elementary school children, the presence of GHD was found in 16 previously unrecognized children out of 555 with short stature $(<3$ rd percentile) and poor growth rates $(<5 \mathrm{~cm} /$ year $)$. Seventeen children were already known to have GHD. Our prevalence number, based on patients referred to paediatric endocrinologists, probably underestimates the true number of GHD children, and a population-based screening is needed to reveal the true prevalence of childhood GHD.

In our series of children with GHD, $41 \%$ were classified as idGHD, $20 \%$ as congGHD and 35\% as acqGHD. The distribution among the three main diagnostic groups of GHD has remained relatively constant over the past 16 years. This is in agreement with the results reported by Cutfield et al. for patients enrolled into KIGS (14). However, the distribution amongst the various aetiologies in our study differs from that reported in other countries or databases. The percentage of idGHD is lower than that observed in other surveys where the percentage of idGHD patients ranges from 65 to $85 \%$ (15-17). In contrast, in our series more patients were diagnosed as congGHD. These discordances can partly be explained by classification differences: in less recent studies, a number of patients with hypothalamo-pituitary malformations who have not been evaluated by a cranial MRI may still have been classified in the idGHD group. Indeed, as the diagnostic procedures progress, more abnormalities by imaging or at the gene level will be found in patients heretofore classified as idGHD. In addition, possible gene defects were not systematically searched for in our idGHD patients. For these reasons, the number of patients classified as idGHD will decrease in the future. Another possible difference between this study and others is the greater proportion of peripubertal patients with constitutional delay of growth and puberty and with low GH secretion included in the other surveys. This latter hypothesis is supported by the finding that the age at onset of rhGH therapy in the idGHD group is lower in Belgium (8.5 years) compared with the patients enrolled, for example, into KIGS (10.3 years) (18).

In line with the data in the literature, boys outnumbered girls in all aetiology groups $(4,15,17,18)$. In patients with idGHD, the ratio of males to females in our study is, however, lower than that reported in other studies (1.5 in Belgium versus 2.5 (USA) (15), 3.1 (Sweden) (17), 1.9 (UK) (17), 2.0 (France) (17), 2.6 (Germany) (17) and 2.0 (KIGS) (18)). This is also the case in patients with acqGHD (1.2 in Belgium versus 2.1 (USA) (15) and 1.5 (KIGS) (18)). In contrast, the ratio of males to females is higher in the Belgian patients with congGHD (3.0 versus 1.3 (USA) (15) and 1.7 (KIGS) (18)) especially in patients with a central malformation that associates an anterior pituitary hypoplasia, a missing, fine or normal pituitary stalk and an ectopic posterior pituitary. In other studies this ratio is not reported.

Although patients with congGHD have GHD from birth, rhGH treatment was started at a mean age of $6.5 \pm 4.7$ years. The delay in starting rhGH therapy may be related to the degree of GH insufficiency associated with the various congenital conditions (19) and/or the delay in diagnosis which depends on the moment of referral to the paediatric endocrinologist. In the idGHD group a bimodal distribution for the age at onset of rhGH therapy is observed with peaks in mid and late childhood. It remains to be shown whether these two cohorts correspond to different pathophysiological mechanisms. In contrast, the age range in the acqGHD group appears broader, from 8 to 15 years. These observations have also been reported by August et al. (15) and by Cutfield et al. for patients in the KIGS database (14). In the patients with idGHD, the age at onset of rhGH therapy ( 8.5 years) is lower than in the patients included in KIGS (10.3 years) (18) or in France (11.1 years) (17) while it is comparable to that of other countries such as the United Kingdom and Germany (8.2 years for the 2 countries) (17). These differences can be due to variations in diagnostic procedures between countries (e.g. sex steroid priming) and/or between the considered time periods in the evaluation of short children for GHD. For the congGHD and acqGHD, the age at onset of rhGH therapy is comparable to other series $(4,15,18)$. The age at onset of rhGH therapy did not change significantly in Belgium between 1986 and 2001 for the three groups. This is in line with the data for the patients with idGHD included in the KIGS database between 1987 and 1997 (16).

In the patients with idGHD and congGHD rhGH treatment was started when a serious height deficit was already present. In patients with idGHD, height at onset of GH therapy ( -2.8 SDS) is comparable to that reported in KIGS $(-2.5$ SDS) (18) but lower than in Sweden for example (-2.3 SDS) (17). In patients with congGHD (-3.0 SDS) and acqGHD ( -1.4 SDS) mean height at onset is comparable to that reported in KIGS (18). In children with idGHD, Cutfield et al. reported a reduction in the height deficit at the start of treatment in patients with idGHD and acqGHD of 0.4 and 0.6 SDS respectively over a period of 10 years (1987-1996) (14). This was not the case in our study.

In line with the data reported in KIGS (18), we observed that the mean birth weight was lower than the population mean in the children with congGHD $(-0.7$ SDS $)$ and idGHD ( -1.0 SDS). These data are in line with data suggesting that $\mathrm{GH}$ plays a role in prenatal growth $(20,21)$. In agreement with the data from the literature $(14,18)$, mean mid-parental height was below the population mean in the patients with idGHD, whereas parental height was normal in the other groups. This finding suggests that patients with familial short stature can present (transient) 
GHD (22), underlining the need for re-evaluation of GH secretion in adulthood (9).

In conclusion, we estimated the prevalence of childhood GHD in Belgium to be 1/5600. Over the past 16 years, the yearly number of new patients and the auxological parameters remained relatively constant. In comparison with the data from other surveys and countries we observed a higher percentage of children with congenital GHD. However, any comparison with other publications is difficult as diagnostic modalities (e.g. MRI, genetic analyses, sex steroid priming) for GHD not only vary between countries, but also between the considered time periods. Moreover, the occurrence of acqGHD in oncologic patients may be related to the tumour treatment which also varies between countries and time periods. Nevertheless, our data underline the need for an accurate cranial MRI evaluation in all children with GHD in order to detect those with central malformations, and the need for genetic analysis in those patients suspected of genetic causes of GHD. As children with congGHD and idGHD are still diagnosed relatively late with a severe height deficit, growth of all children, especially those with short stature, should be monitored very carefully, and timely referral to a paediatric endocrinologist with experience in growth disorders should be considered.

\section{Acknowledgements}

We are grateful to the other members of the Belgian Study Group for Paediatric Endocrinology who took part in this work: V Beauloye, D Beckers, L Dooms, I François, M C Lebrethon, P Malvaux, R Rooman, $\mathrm{S}$ Tenoutasse, J Vanbesien, $\mathrm{M}$ Vandeweghe and $\mathrm{M}$ van Helvoirt. This work was supported by grants from the Foundation of the Belgian Study Group for Paediatric Endocrinology.

\section{References}

1 Lindsay R, Feldkamp M, Harris D, Robertson J \& Rallison M. Utah Growth Study: Growth standards and the prevalence of growth hormone deficiency. Journal of Pediatrics $199412529-35$.

2 Vimpani GV, Vimpani AF, Lidgard GP, Cameron EH \& Farquhar JW. Prevalence of severe growth hormone deficiency. British Medical Journal $197713427-430$.

3 Drent ML, Delemarre-Van de Waal HA \& Wit JM. Eenmaal groeihormoon, altijd groeihormoon? De transitie van groeihormoonbehandeling van kind naar volwassenen. Nederlands Tijdschrift voor Geneeskunde 2002146 154-157.

4 Werther G. Growth hormone measurements versus auxology in treatment decisions: the Australian experience. Journal of Pediatrics $1996 \mathbf{1 2 8}$ S47-S51.

5 Tani N. Epidemiological study of dwarfism in Nigata prefecture. Folia Endocrinologica Japonica 198561 1295-1309.

6 Bao XL, Shi YF, Du YC, Liu R, Deng JY \& Gao SM. Prevalence of growth hormone deficiency of children in Beijing. China Medical Journal $1992 \mathbf{1 0 5} 401-405$.
7 Parkin JM. Incidence of growth hormone deficiency. Archives of Disease in Childhood 197449 904-905.

8 Audi L, Gilabert A, Lloveras G, Marti-Henneberg C, RodriguezHierro F, Vilardell E et al. Long-term GH therapy: epidemiology and auxologic outcome. Hormone Research 2002 57 113-119.

9 Thomas M, Massa G, Bourguignon JP, Craen M, De Schepper J, de Zegher F et al. Growth hormone $(\mathrm{GH})$ secretion in patients with childhood-onset GH deficiency: retesting after one year of therapy and at final height. Hormone Research 200359 7-15.

10 Ranke MB. The KIGS aetiology classification system. In Growth Hormone Therapy in KIGS - 10 Years Experience, pp 389-401. Eds MB Ranke \& P Wilton. JA Barth Verlag; Heidelberg, Leipzig, 1999.

11 Tanner JM, Whitehouse RH \& Takaishi M. Standards from birth to maturity for height, weight, height velocity and weight velocity: British children 1965. Archives of Disease in Childhood 196641 454-471, 613-635.

12 Niklasson A, Ericson A, Fryer JG, Karlberg J, Lawtrence C \& Karlberg P. An update of the Swedish references standards for weight, length and head circumference at birth for given gestational age (1977-1981). Acta Paediatrica Scandinavica 199180 756-762.

13 Population et ménages. In Population Totale et Belge au 1.1.2001, pp 38. Ed Institut National de Statistique, Bruxelles, 2001.

14 Cutfield WS, Jönsson P \& Bagewitz A. Demographic trends: 1987-1996. In Biannual Report no. 15, pp 3-12. Stockholm: Pharmacia and Upjohn International Growth Database, 1997.

15 August GP, Lippe BM, Blethen SL, Rosenfeld RG, Seelig SA, Johanson AJ et al. Growth hormone treatment in the United States: demographic and diagnostic features of 2331 children. Journal of Pediatrics $1990 \mathbf{1 1 6} 899-903$.

16 Chatelain P. Trends in the diagnosis and treatment of short stature as revealed by KIGS. In Growth Hormone Therapy in KIGS 10 Years Experience, pp 11-20. Eds MB Ranke \& P Wilton. JA Barth Verlag; Heidelberg, Leipzig, 1999.

17 Price DA, Johnston Betts PR, Buckler JM \& Donaldson MD. Biosynthetic human growth hormone treatment in the UK: an audit of current practice. Archives of Disease in Childhood 1994 $71266-271$.

18 Ranke MB. Lindberg A on behalf of the KIGS International Board. Demographic characteristics of patients within KIGS: special emphasis on sex and onset of puberty. In Biannual Report no. 18, pp 1-9. Stockholm: Pharmacia International Database, 2001.

19 Miyamoto J, Hasegawa Y, Ohnami N, Onigata K, Kinoshita E, Nishi Y et al. Development of growth hormone and adrenocorticotropic hormone deficiencies in patients with prenatal or perinatal onset hypothalamic hypopituitarism having invisible or thin pituitary stalk on magnetic resonance imaging. Endocrine Journal $200148355-362$.

20 De Luca F, Bernasconi S, Blandino A, Cavallo L \& Cisternino M. Auxological, clinical and neuroradiological findings in infants with early onset growth hormone deficiency. Acta Paediatrica $199584561-565$

21 Gluckman PD, Gunn AJ, Wray A, Cutfield WS, Chatelain PG, Guilbaud $\mathrm{O}$ et al. Congenital idiopathic growth hormone deficiency associated with prenatal and early postnatal growth failure. The International Board of the Kabi Pharmacia International Growth Study. Journal of Pediatrics $1992121920-923$.

22 Pozo J, Argente J, Barrios V, Gonzalez-Parra S, Munoz MT \& Hernandez H. Growth hormone secretion in children with normal variants of short stature. Hormone Research $1994 \mathbf{4 1}$ 185-192.

Received 10 December 2003

Accepted 6 April 2004 